\title{
The Control \& Housekeeping system of the High Energy Particle Detector and its performances during 3 years of flight on board CSES
}

\section{De Donato Cinzia ${ }^{a, *}$ and Masciantonio Giuseppe ${ }^{a}$ on behalf of the CSES-Limadou Collaboration}

(a complete list of authors can be found at the end of the proceedings)

aIstituto Nazionale di Fisica Nucleare (INFN) Sezione di Roma "Tor Vergata", via della Ricerca Scientifica 1,00133 Roma (RM), Italy

E-mail: cinzia.dedonato@roma2.infn.it, giuseppe.masciantonio@roma2.infn.it

The China Seismo-Electromagnetic Satellite is a multi-instrument space mission dedicated to the investigation of the topside ionosphere structure and dynamics (plasma parameters, electromagnetic fields and charge particles fluxes) and the possible correlation of its perturbations with the occurrence of high magnitude earthquakes. The main contribution of the Italian collaboration to the mission is the High Energy Particle Detector (HEPD), designed and built for the detection of electrons and protons in the energy range 3-100 MeV and 30-200 MeV, respectively. The satellite was launched on February 2, 2018 from the Jiuquan Satellite Launch Center (Inner Mongolia, China) and HEPD is fully operational since July 28, 2018. To ensure correct operations and optimal performances during the expected life time of 5 years, the HEPD on-board software hosts the Control \& Housekeeping system responsible for the detector management and monitoring. The system handles instrument data acquisition and calibrations, HEPD configuration and monitoring and acts as the main interface of the detector with the satellite platform. The continuous monitoring of HEPD status allows to control the detector functionality, to check electronics stability, to identify anomalous behaviors and to perform recovery actions if necessary. Besides, the high configurability of the detector allows to modify HEPD configuration in order to preserve its detection efficiency that can deteriorate along with the detector age. In this paper we describe the HEPD Control \& Housekeeping system and HEPD operational status during its 3 years of flight.

$37^{\text {th }}$ International Cosmic Ray Conference (ICRC 2021)

July 12 th - 23rd, 2021

Online - Berlin, Germany

\footnotetext{
*Presenter
} 


\section{Introduction}

The China Seismo-Electromagnetic Satellite (CSES) is a multi-payload scientific mission dedicated to study electromagnetic, plasma and particle perturbations of atmosphere, ionosphere, magnetosphere and Van Allen belts induced by natural sources and anthropocentric emitters and their potential correlations with the occurrence of seismic events [1]. The satellite has two distinct working regions, a payload working zone in the geographical latitude range between $-65^{\circ}$ and $+65^{\circ}$, where data are collected and transmitted, and a STANDBY zone at latitudes $>+65^{\circ}$ or $<-65^{\circ}$, where satellite operations, such as solar panel rotation or attitude adjustments, are performed and all the payloads are switched to minimum functionality and data are not acquired.

Among the payloads on-board CSES, the Italian CSES-Limadou collaboration developed the HighEnergy Particle Detector (HEPD), devoted to the observation of electrons and protons in the range 3-100 MeV and 30-200 MeV, respectively [2]. The satellite was launched on February 2, 2018 from the Jiuquan Satellite Launch Center (Inner Mongolia, China) and HEPD is fully operational for regular data taking since July 28, 2018 [3]. To ensure correct operations and optimal performances during the expected life time of 5 years, the HEPD on-board software hosts the Control \& Housekeeping $(\mathrm{C} \& \mathrm{H})$ system responsible for the detector management and monitoring, as well as for its communications with the satellite platform via CAN-bus interface. A description of the HEPD instrument and of the C\&H system is given in $\$ 2, \S 3$ while the HEPD in-flight operations and monitored status during its first 3 years of flight are described in $\S 4, \S 5$.

\section{HEPD instrument}

The HEPD consists of different subsystems, the detector, the power supply, the electronics (ELS) and the mechanics. The detector is designed to identify and measure energy and direction of electrons and protons crossing the instrument [2,3]; starting from the aperture window (Fig.1a), it comprises two double-sided silicon microstrip planes providing the incident particle direction, one thin segmented layer of plastic scintillators for the trigger signal generation, and a calorimeter, constituted by a tower of 16 plastic scintillator counters and a $3 \times 3$ matrix of LYSO inorganic scintillator crystals, for energy measurements; five plastic scintillator planes surrounding the calorimeter (Veto) are used to reject particles that enter the apparatus from outside the acceptance window and those that are not fully contained within the calorimeter. For all scintillators readout, R9880U-210 Hamamatsu PMTs are used.

The power supply subsystem includes a Low-Voltage Power Supply (LVPS) and a High-Voltage Power Supply (HVPS); the LVPS generates two low-voltage power lines (5.6V and 3.6V) for the electronic subsystem from the satellite power bus (29 V); the HVPS consists of 12 HV step-up modules that produce high-voltage lines for the Silicon sensors and PMTs bias.

The ELS (Fig.1b) is composed by all front-end electronics and five boards [4]: the TM/TC Power Control board, responsible for the LVPS management and voltages distribution towards electronics and hosting a direct link to the satellite for local TeleMetry and direct TeleCommand (wired TM/TC), the HV Control board managing the $12 \mathrm{HV}$ step-up modules, the TRIGGER board, responsible for the Trigger System management and PMT data acquisition, the DAQ board, responsible for Silicon data acquisition/processing and for the management of the scientific data 


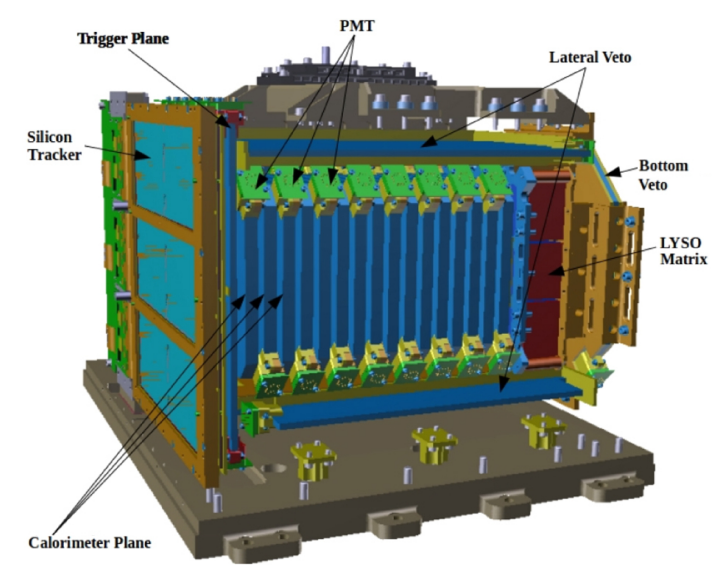

(a) High Energy Particle Detector (HEPD); one lateral Veto plane and box panels have been removed for visualization purposes.

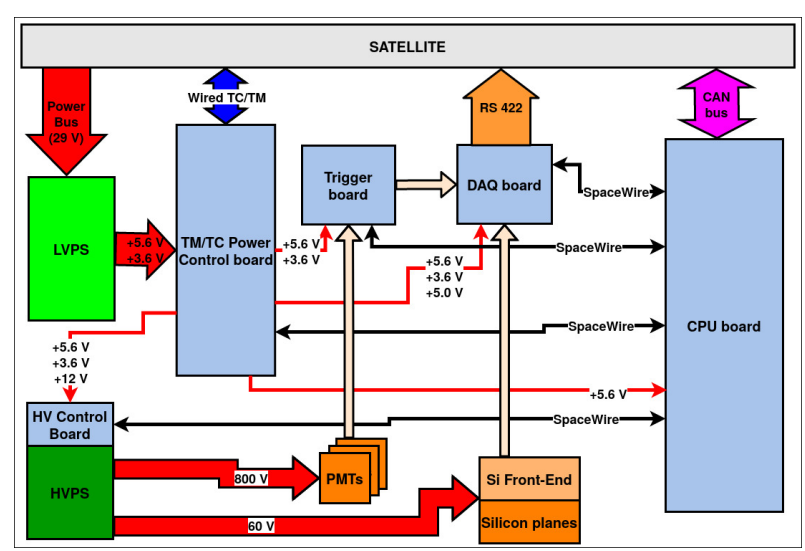

(b) Schematics of HEPD electronic and power supply subsystems; communication and power lines between the boards and toward the satellite are shown as well.

Figure 1: The High Energy Particle Detector (HEPD)

formatting and transmission to the satellite via the RS422 bus [5], and the CPU board hosting the Control \& Housekeeping system responsible for the configuration, management and monitoring of the whole detector and for the communication with the platform of the satellite via the CAN-bus interface. To avoid permanent failures, each electronic boards and power supply is duplicated in a MAIN and SPARE side for redundancy.

A schematic of HEPD electronic and power supply subsystems is shown in Fig.1b together with the communication and power lines between the boards and toward the satellite.

\section{Control \& Housekeeping system}

The C\&H system manages the bi-directional CAN-bus interface allowing to receive both satellite broadcasts and TeleCommands (CAN-bus TCs) and to send back telecommand replies (TC acknowledge) and payload TeleMetry (CAN-bus TM) containing information about the detector status. TCs are used to manage and configure HEPD while satellite broadcasts are messages periodically sent by the satellite to all payloads providing information about position, velocity, time and attitude of the satellite itself; Fast and Slow TM data are requested periodically (with a cycle of $1 \mathrm{~s}$ and $8 \mathrm{~s}$, respectively) by the satellite in order to collect the status of the payload.

The $\mathrm{C} \& \mathrm{H}$ system is responsible for the management of detector operations as well as for diagnostic routines providing information about the detector status, by means of the internal Slow Control link (SpaceWire link) that allows communications between CPU and the other ELS boards. The management of the HEPD operations includes temporal tag of runs and broadcast (local time, absolute time) and the storage of non volatile information.

For reliability, the C\&H system is implemented in an application program stored in two non-volatile memory chips of CPU board: a writable FRAM and a read-only flash memory (EEPROM). The main program routines are performed by a DSP while the FPGA is responsible of the management of SpaceWire and CAN-bus interfaces, of the DSP boot control and DATA memories $[4,5]$. 
At power-on the Low-Voltage Control board enables the voltage towards the CPU board and the application program and relative data (such as previous boot status and configuration) are uploaded on the DSP. After CPU boot, the C\&H system is in charge of the power-on and initialization of HEPD subsystems, following a defined sequence and checking the basic functionalities of each board. If all boards are powered-on correctly, HEPD is set in SAFE or NOMINAL mode depending on the saved configuration; in SAFE mode, used mainly for tests, no voltages are applied to the sensors, while in NOMINAL mode the HVPSs are powered at their nominal values providing bias to PMTs and Silicon ladders. The instrument power-off and the transition between OPERATIONAL
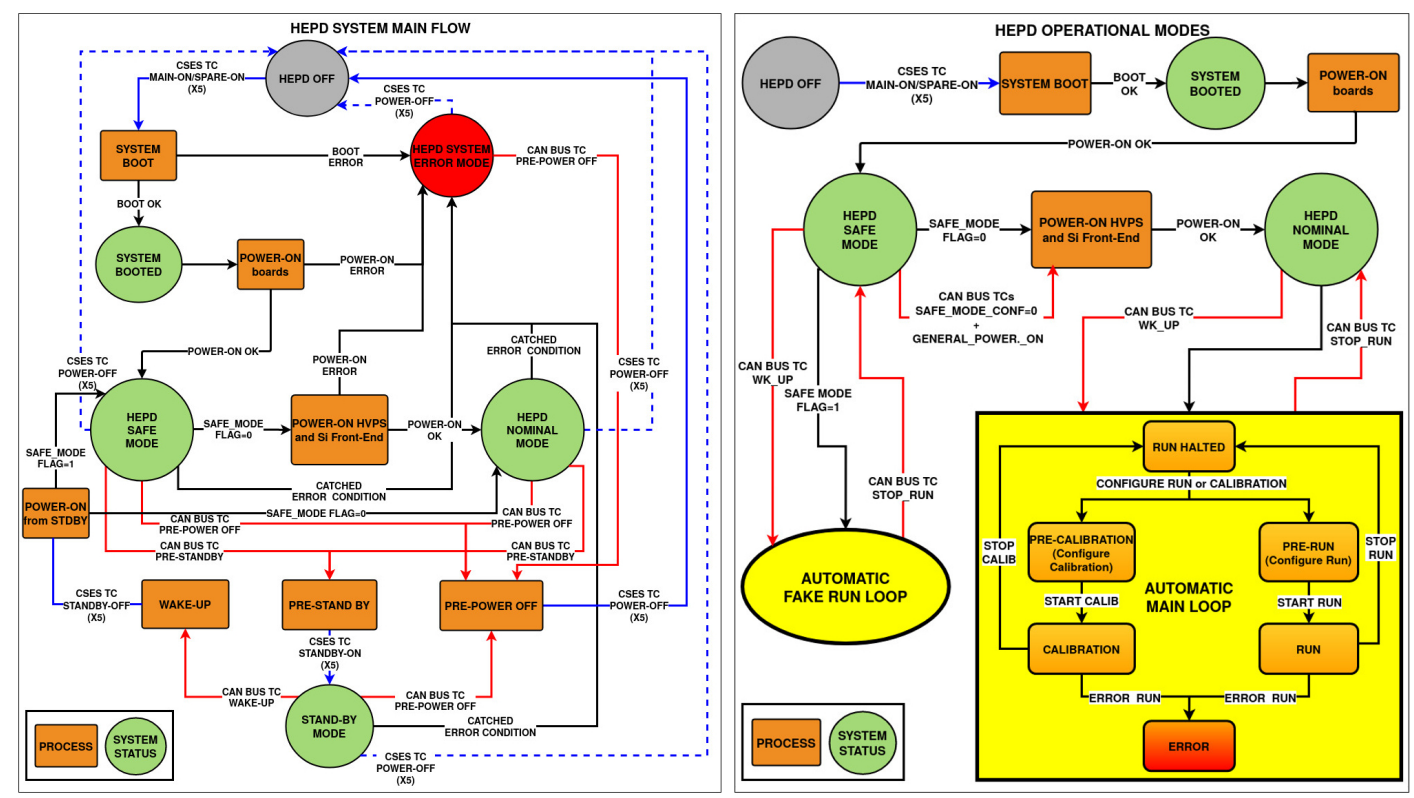

Figure 2: $L E F T$ - HEPD Main processes and states are shown together with the main commands for state transitions. RIGHT - Operational modes and the main automatic loop are shown along with the required telecommands and configuration parameters for transition between safe and nominal mode. Wired commands and CAN-bus telecommands are marked by blue and red arrows, respectively.

modes (SAFE/NOMINAL) and STAND-BY mode (non acquisition zones) are made by means of dedicated wired and CAN-bus TCs managed by the TM/TC Power Control board and C\&H system, respectively, as shown in Fig.2 (left).

Once set in OPERATIONAL mode, the system configures and executes data acquisition and calibration automatically inside a main automatic loop (Fig.2 right); in particular the system recognizes the orbital zone from satellite broadcasts and configures acquisition/calibration sending the correspondent orbital configuration to the Trigger board and DAQ board via Slow Control link; non volatile information as Boot Number and HEPD status before and after boots are stored in FRAM while auxiliary information as broadcast data, timing information and current HEPD configuration are sent to the DAQ in order to be included in the scientific data output.

During operations, the $\mathrm{C} \& \mathrm{H}$ system periodically monitors all electronic subsystems via Slow Control link and sends TM data containing diagnostic information to the satellite via CAN-bus link; the acquired telemetry, transmitted from the satellite to the ground stations once a day, includes the monitored status and error registers of each subsystem, temperatures, the monitored HV values 
and LV power goods. Other housekeeping information relevant for the good functionality of the detector is sent to the DAQ in order to be included in the scientific data [4]. In particular, the 63 single PMT rate meters provided by the Trigger board are used to identify (and eventually mask) broken or noisy PMTs that could affect the Trigger system efficiency.

\section{In-flight operations}

The China Seismo Electromagnetic Satellite (CSES) was launched on February 2, 2018 from the Jiuquan Satellite Launch Center (JSLC) near Jiuquan, Gansu Province (China).

HEPD on board operations begun on February 6, 2018 with the payload "Health check" procedures dedicated to testing all the electronics and satellite interfaces, as well as the general functionality of the detector, using the real-time Telemetry data provided by the $\mathrm{C} \& \mathrm{H}$ system.

In the following months the HEPD underwent the Commissioning phase, ended on July 28, 2018, devoted to the definition of the optimal in-flight detector configuration (PMT threshold values and HEPD trigger configuration for the generation of the trigger signal, HV values for PMTs bias, orbital zones definition, etc...). During this phase the stability of the electronics and of the whole apparatus has been checked and the operational parameters of the instrument were optimized implementing several on-board configurations (thanks to the high configurability of the detector by means of CAN-bus TCs) and analyzing the correspondent HEPD performances [3, 4].

At the end of the commissioning phase, the final optimal configuration was fixed and written on CPU FRAMs and HEPD has been set for regular data-taking mode: the payload is set in NOMINAL mode and executes runs/calibrations automatically.

Up to day, post-commissioning operations have been mainly performed in order to power-off HEPD for satellite maneuvers or temperature issues (standard procedure), to reconfigure the detector and to recover HEPD from anomalous behaviors, identified by means of TM data (see Section 5.1). For most of the anomalies detected until now, HEPD functionality was recovered automatically or by the application of the power-off procedure and reboot. However special specific procedures have been necessary in order to restore HEPD functionality for two anomalies involving the corruption of the configuration written on FRAM and to modify HEPD configuration (extension of the acquisition zone to higher latitudes, in accordance with satellite managers).

\section{Post Commissioning In-flight Status}

From the end of the commissioning operations up to April 14, 2021, HEPD has collected about 14000 hours of acquisition over about 21000 hours of flight. The useful time for acquisition of scientific data has been limited from one side by the duty time due to the high-latitude standby zones, from the other side by HEPD malfunctions and operations. Considering only the time spent in acquisition zones, the HEPD has been able to acquire scientific data for about $89.45 \%$ of the time, for $64 \%$ of time on the MAIN side and $25.5 \%$ on SPARE side (Fig.3), while for the remaining $10.55 \%$ of time ("acquisition downtime") the HEPD has not been able to acquire scientific data for:

- $1.4 \%$ Regular reboots agreed with satellite managers for HEPD reconfiguration, satellite maneuvers, temperature issue and other normal power-off: contributions of different cases are given in Fig.3 (right); 
- 9.2\% Detected anomalies (malfunctions) and related recovery actions (see 5.1).

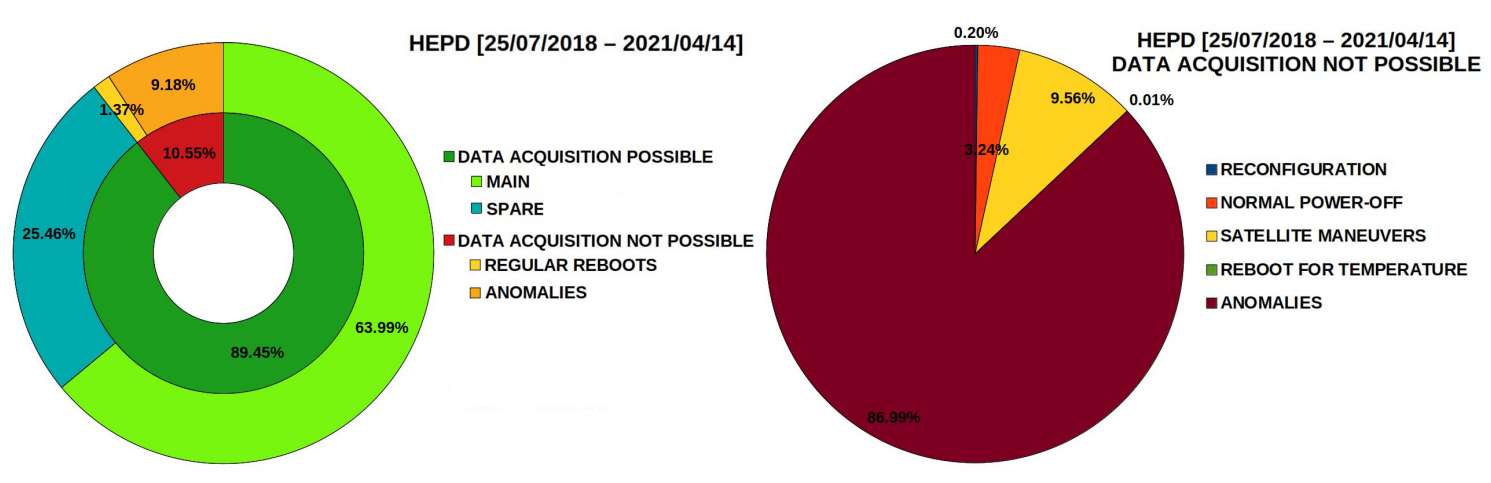

Figure 3: HEPD Post-commissioning phase acquisition time [25/07/2018-14/04/2021]. LEFT-Estimation of HEPD acquisition time and downtime; information is given for each side (MAIN/SPARE) for data acquisition, overall for regular reboots and anomalies. RIGHT - Contribution of anomalies and different cases of regular reboots to the acquisition downtime.

A more careful monitoring of the HEPD status allowed to reduce progressively the "acquisition downtime" which is strongly related to the prompt recognition of anomalous behaviors and on the scheduling of satellite operations (described in §4). For example the "downtime" amounted to about $23 \%$ of its lifetime during the first year of post-commissioning phase, decreasing to about $6.6 \%$ in the following period (about 1 year and 9 months).

\subsection{Housekeeping data}

The Telemetry data provided by the $\mathrm{C} \& \mathrm{H}$ system allow to check the stability of the electronics, to identify anomalous behaviors and to investigate the possible source of malfunctions. In particular, the detailed analysis of telemetry data (fast, slow and wired TM) allowed to detect and identify various anomalies in HEPD functionality, most of which can be classified as:

- Partial/Total power-off of HEPD electronics (or failed reboot of one ELS) due to LVPS issue: most of these observed anomalies can be related to a common problem/cause interesting the output section of the main LVPS $(5.6 \mathrm{~V})$ or to fast transient of current/voltage in the LVPS that could be not detectable in telemetry;

- Anomalies related to Radiation issue: some encountered malfunctions seemed to be related to radiation; in particular, in two cases a SEU (Single Event Upset) ${ }^{1}$ could be the responsible of the corruption of the HEPD configuration written on the CPU FRAM.

Among the housekeeping data, an important parameter is the temperature of the system, recorded both in TM data and in Scientific Run data. An abnormal increase of the temperature can indicate or can be the cause of a malfunction of the apparatus; because of such increases, the HEPD has been reboot few times according with satellite managers, in order to keep the instrument in its working range of temperatures. The temperatures of CPU and Trigger boards and of satellite baseplate from

\footnotetext{
${ }^{1}$ Single Event Upset may be responsible of single/multiple bit changes or corruption of a local zone of memory (program/configuration) with consequent read/write of wrong register address or register content.
} 


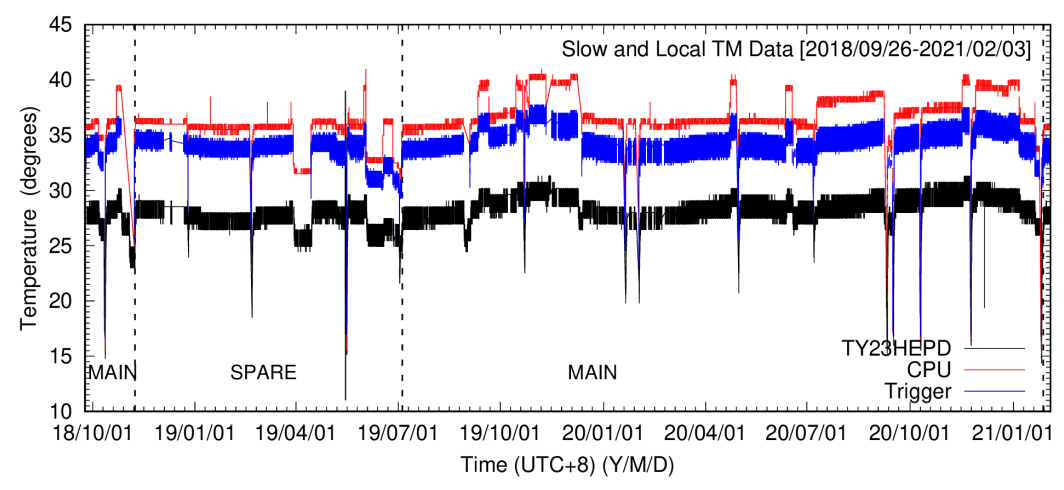

Figure 4: HEPD temperatures [2018/09/27-2021/02/03]: CPU and Trigger board temperatures (from Slow TM data) and baseplate temperature (TY23HEPD, from Local wired Telemetry) are shown.

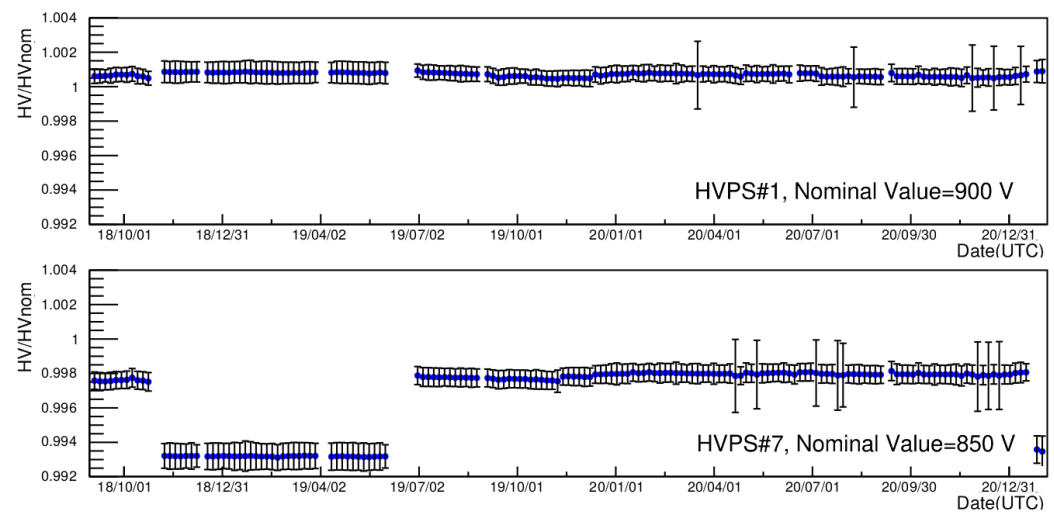

Figure 5: Monitored $H V$ values with respect to the nominal one for main/spare sides for two HVPSs set at different nominal values: the variations of monitored $H V$ values with respect to the nominal one are averaged over 5 days intervals and shown for the period [2018/09/05-2021/02/03].

the end of September 2018 to the beginning of February 2021 are shown in Fig.4. The analysis of TM data revealed that temperature spikes interest mainly the MAIN side and are often correlated with operational malfunctions.

Available TM data have been also fully analyzed to check electronics stability, such as the HVPS voltages, one of the parameters contributing to the stability of the instrument's performance efficiency. Other parameters (PMT pedestals and rate meters and HEPD response to MIP2) are periodically monitored using Scientific data [4]. In Fig.5 the variation of monitored HV values with respect to the nominal one over the period September 2018 - February 2021 is given for two HVPSs biasing PMTs set at different nominal values. In the plot, the HV monitored values have been averaged over 5 days intervals and compared with the nominal one; the point error bars represent the standard deviation of the HV distribution in order to highlight the dispersion of the HV monitored data over the 5 days intervals. Despite the visible discontinuity due to the switch from the MAIN to the SPARE side of the electronics, the HVPSs show a very stable behavior with an overall variation below $0.75 \%$ for HVPSs biasing PMTs and below $1.5 \%$ for those biasing the

${ }^{2}$ Minimum Ionizing Particle 
Silicon sensors.

\section{Conclusion}

The HEPD was launched on-board CSES on February 2, 2018 from the Jiuquan Satellite Launch Center (Inner Mongolia, China) and, since July 28, 2018 it is fully operational for regular data taking. The $\mathrm{C} \& \mathrm{H}$ system, responsible for the detector management and monitoring and for the communication with satellite via CAN-bus link, has been designed to ensured correct operations and optimal performances during the expected life time of 5 years. Since first flight phases, the system demonstrated to handle correctly detector activities and operations, allowing several changes of configuration and to perform recovery actions in case of anomalous behaviours. Malfunctions and relative origins were identified by means of TM data, provided by the continuous monitoring of the apparatus carried out by the system; nevertheless more diagnostic information at electronic level would be needed to investigate deeply the causes. For the improved version of HEPD detector on-board CSES-02 satellite (HEPD-02), currently under development, the C\&H system will be upgraded with more diagnostics (monitored parameters and automatic recovery actions) and operational functions.

\section{Acknowledgments}

This work was supported by the Italian Space Agency in the framework of "Accordo Quadro n.2017-19-H.0 per LIMADOU fase E/Operazioni", (CUP F82F17000320005) and "Accordo Attuativo n. 2021-1-HH.0 della Convenzione Quadro n. 2016-4- Q.0" per "Limadou Operazioni-FO", (CUP F19C21000140005).

\section{References}

[1] X. Shen, et al., The state-of-art of the China Seismo-Electromagnetic Satellite mission, Sci. China Technol. Sci., vol. 61, no. 5 (2018) pp.634-642.

[2] G. Ambrosi, et al., The HEPD particle detector of the CSES satellite mission for investigating seismo-associated perturbations of the Van Allen belts, Sci. China Technol. Sci., vol. 61, no. 5 (2018) pp.643-652.

[3] P. Picozza, et al., Scientific Goals and In-orbit Performance of the High-energy Particle Detector on Board the CSES, ApJS vol. 243 (2019) pp. 16.

[4] G. Ambrosi, et al., The electronics of the High-Energy Particle Detector on board the CSES satellite Nuclear Inst. and Methods in Physics Research, A (2021), doi: https://doi.org/10.1016/j.nima.2021.165639.

[5] A. Sotgiu, et al., Control and Data Acquisition Software of the High-Energy Particle Detector on board the CSES Space Mission, Softw: Pract Exper. vol.51, issue 6 (2021) pp.1459-1480. 


\section{Full Authors List: CSES-Limadou Collaboration}

S. Bartocci ${ }^{1}$, R. Battiston ${ }^{2,3}$, F. Benotto ${ }^{4}$, S. Beole ${ }^{4,5}$, W.J. Burger ${ }^{3,6}$, D. Campana ${ }^{7}$, G. Castellini ${ }^{8}$, P. Cipollone ${ }^{1}$, S. Coli ${ }^{4}$, L. Conti ${ }^{1,9}$, A. Contin ${ }^{10,11}$, M. Cristoforetti ${ }^{12}$, L. De Cilladi ${ }^{4,5}$, C. De Donato ${ }^{1}$, C. De Santis ${ }^{1}$, F.M. Follega ${ }^{2,3}$, G. Gebbia ${ }^{2,3}$, R. Iuppa ${ }^{2,3}$, M. Lolli $^{11}$, N. Marcelli $i^{1,13}$, M. Martucci ${ }^{1,13}$, G. Masciantonio ${ }^{1}$, M. Mergé ${ }^{1, \dagger}$, M. Mese ${ }^{7,14}$, C. Neubuser ${ }^{3}$, F. Nozzoli ${ }^{3}$, A. Oliva ${ }^{11}$, G. Osteria $^{7}$, L. Pacini ${ }^{15}$, F. Palma ${ }^{1, \dagger}$, F. Palmonari ${ }^{10,11}$, A. Parmentier ${ }^{1}$, F. Perfetto ${ }^{7}$, P. Picozza ${ }^{1,13}$, M. Piersanti ${ }^{16}$, M. Pozzato ${ }^{11}$, E.

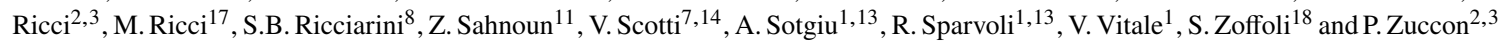

${ }^{1}$ INFN-Sezione di Roma "Tor Vergata”, V. della Ricerca Scientifica 1, I-00133 Rome, Italy;

${ }^{2}$ University of Trento, V. Sommarive 14, I-38123 Povo (Trento), Italy;

${ }^{3}$ INFN-TIFPA, V. Sommarive 14, I-38123 Povo (Trento), Italy;

${ }^{4}$ INFN-Sezione di Torino, Via P. Giuria 1, I-10125 Torino, Italy;

${ }^{5}$ University of Torino, Via P. Giuria 1, I-10125 Torino, Italy;

${ }^{6}$ Centro Fermi, V. Panisperna 89a, I-00184 Rome, Italy;

${ }^{7}$ INFN-Sezione di Napoli, V. Cintia, I-80126 Naples, Italy;

${ }^{8}$ IFAC-CNR, V. Madonna del Piano 10, I-50019 Sesto Fiorentino (Florence), Italy;

${ }^{9}$ Uninettuno University, C.so V. Emanuele II 39, I-00186 Rome, Italy;

${ }^{10}$ University of Bologna, V.le C. Berti Pichat 6/2, I-40127 Bologna, Italy;

${ }^{11}$ INFN-Sezione di Bologna, V.le C. Berti Pichat 6/2, I-40127 Bologna, Italy;

${ }^{12}$ Fondazione Bruno Kessler, V. Sommarive 18, I-38123 Povo (Trento), Italy;

${ }^{13}$ University of Rome "Tor Vergata", V. della Ricerca Scientifica 1, I-00133 Rome, Italy;

${ }^{14}$ University of Naples "Federico II", V. Cintia 21, I-80126 Naples, Italy;

${ }^{15}$ INFN-Sezione di Firenze, V. Sansone 1, I-50019 Sesto Fiorentino (Florence), Italy;

${ }^{16}$ INAF-IAPS, V. Fosso del Cavaliere 100, I-00133 Rome, Italy;

${ }^{17}$ INFN-LNF, V. E. Fermi 54, I-00044 Frascati (Rome), Italy;

${ }^{18}$ Italian Space Agency, V. del Politecnico, I-00133 Rome, Italy;

$\dagger$ At ASI Space Science Data Center (SSDC) also, V. del Politecnico, I-00133 Rome, Italy. 